\title{
Téoros
}

Revue de recherche en tourisme

\section{L.J. Rivet : un pionnier de l'industrie touristique du Québec}

\section{Pierre Mayrand}

Volume 14, numéro 2, été 1995

Le tourisme : toute une histoire!

URI : https://id.erudit.org/iderudit/1075098ar

DOI : https://doi.org/10.7202/1075098ar

Aller au sommaire du numéro

Éditeur(s)

Université du Québec à Montréal

ISSN

0712-8657 (imprimé)

1923-2705 (numérique)

Découvrir la revue

Citer cet article

Mayrand, P. (1995). L.J. Rivet : un pionnier de l'industrie touristique du Québec. Téoros, 14(2), 26-29. https://doi.org/10.7202/1075098ar d'utilisation que vous pouvez consulter en ligne.

https://apropos.erudit.org/fr/usagers/politique-dutilisation/ 


\title{
L.J. Rivet : un pionnier de I'industrie touristique du Québec
}

\author{
Pierre Mayrand ${ }^{*}$
}

Lors de la création du Centre d'étudesdu tourismeà l'UQAM, sous l'impulsion de P.-O. Courtemanche, et de l'institution des Journées québécoises du tourisme culturel, en collaboration avec Louis Jolin, et enfin de l'Association technique du tourisme (premier regroupement de professionnels du tourisme), il apparut rapidement qu'il serait utile d'entreprendre et de diffuser des recherches sur les origines de l'industrie touristique au Québec, dans une perspective culturelle ouverte par la $D \hat{e}$ claration québécoise du tourimue culturel. C'est ainsi que naquit, en 1978, au sein du Groupe de recherche en patrimoine de l'UQAM, une première étude sur la première agence de voyages et la première publication touristique au Québec, mise sur pied par Louis Joseph Rivet, à partir de 1901, jusqu à son association avec l'un des grands du tourisme québécois moderne, soit Jules Hone vers 1911, et la première édition du Touriste en 1912. Cette étude publiée dans Documents: Patrimoine et arts anciens du Québec, en septembre 1978, devait être suivie d'autres études et d'autres projets de diffusion auprès des intervenants touristiques. Seuls paraitront, Les lieux et l'architecture de villégiature au Québec: histoire et phénomène $(1985)^{(1)}$ et plusieurs événements muséologiques organisés par le Groupe de recherche en patrimoine autour du thème Le voyage en folie $(1985)^{(2)}$. Nous avons pensé que les textes de base de la publication restreinte de 1978 étaient suffisamment instructifs pour en proposer la reproduction intégrale dans la présente édition de Téoros. Nous ne pouvons que souhaiter une reprise des recherches selon l'approche qui fut la nôtre il $y$ a dix-sept ans: *Il faut souhaiter que cette modeste contribution à la connaissance de notre patrimoine touristique témoignera de l'importance accordée aux aspects culturels du tourisme dès l'origine,

- Monsieur Pierre Mayrand est membre fondateur
du Centre d' études du tourisme et professeur en
patrimoine et musbologie a l'Universite du Quebec a Mantréal. de même qu'à ceux de l'entreprise touristique, pour qu'ainsi, nous puissions nous inspirer de ces attitudes dans l'adoption de moyens pour améliorer la qualité des voyages... celle-ci se voulant avant tout, humaine et créatrices (Groupe de recherche en patrimoine, document no 5 , septembre 1978, remerciements).

\section{Louis Joseph Rivet : témoin d'une époque du voyage au Québec}

Le tourisme n'est pas un phênomène rếcent au Québec. II remonte, dans sa forme organisée, au début du XIX ${ }^{e}$ siècle. Il est d'abord constitué presque exclusivement par le tourisme réceptif, composé en grande partie, d'une clientële américaine. L'attrait touristique du Haut et du Bas-Canada - la pénétration se faisant par le Richelieu et les principales voies de bifurcation vers l'Est (Québec et sa région: Kamouraska en aval et Pointe-au-Pic) et vers l'Ouest (Mille Iles et Lac Ontario) - se faisant à partir de Montréal, d'où la concentration dans cette ville de nombreuses entreprises de navigation.

La poussée américaine, la formation d'une bourgeoisie canadienne, le perfectionnement des moyens de transport (MontuéalQuébec en 24 heures, par bateau) contribuent à la création, très tôt, de sites de villégiatures rếputés, tel que Cacouna. $\mathrm{La}$ renomméedecessites, tant pour leur beauté que pour la société qui en fera ses lieux d'élection, de même que les particularités de la paysannerie canadienne-française (faclité de services, traditions et langue) entraînent une multiplication étonnante de manuels et autres formes de guides touristiques dans lesquels sont décrits les sites et monuments, de même que sont proposés des circuits et présentées les conditions de séjour et les installations d'accueil faisant ainsi la promotion des entreprises.

La création de réflexes touristiques au Québec et d'une tradition d'affaires pour les agences qui les alimentent, se convertiront en une vogue pour le tourisme à l'étranger aussitôt que des moyens sécuritaires de navigation apparaittront. Cette transition correspond également à la richesse des villes, à la levée des blocus, à l'avènement de l'immigration massive (on transportait les touristes et l'on ramenait des immigrants) et à l'ouverture internationale du Canada trouvant sa première identité lors du pacte de la Confédération.

Le rétablissement de relations soutenues avec le clergé et avec les grandes capitales chrétiennes du monde (Paris et Rome) liens qui se traduisent également dans l'architecture et le décor (églises de Bourgeault, décor du château Dufresne, à Montréal, décor peint et verrières des églises...) de même que l'apaisement du Moyen-Orient(Terre-Sainte), conduiront de plus en plus de Québécois (clientèle cléricale et son entourage) à entreprendre un voyageà l'étranger. Alorsque le tourisme de villégiature de type réceptif ou indigène commence à décliner au début du $\mathrm{XX}^{2}$ siècle - la disparition progressive des guides en témoigne - le voyageà l'érranger prend de plus en plus de place.

C'est dans ce contexte que naissent les entreprises de Louis Joseph Rivet puis de Jules Hone. L'originalité de celles-ci est liée à leur caractère francophone et aux moyens qu'elles adoptent afin de se faire connaître. Le rổle culturel joué par ces agences est important: elles diffusent dans le public, soit par la Presse, soit par leurs dépliants, une information articulée sur les pays de destination, contribuant ainsi à une plus grande ouverture du grand public Québécoisverslesréalités étuangères. Cette information complète ainsi l'éducation populaire transmise à l'occasion des foires saisonnières (cirques, théâtres...) et des expositions universelles a lors très en vogue à cette époque.

On peut émettre l'hypothèse, également, que l'action des entreprises Rivet et Hone aura contribuéa à l'éclatement des privilèges de voyage - réservés jusqu'au XX* siêcle auxclasses aisées (commerçants, industriels, entrepreneurs de voyage étrangers, issus de milieux anglophones pour la plupart) et leurs accessibilités aux classes moyennes 
et rurales par le biaisdespèlerinages, worme particulière de tourismes comme le décrit Roger Brière.

L'utilisation des mass média comme instrumentde promotion touristique, la forme dela publicitéqui emprunte un caractère et un dynamisme qu'on lui retrouve encore aujourd'hui (chronique du voyage, format du dépliant, annonces publicitaires, recherche de tarifs adaptés à différentes bourses, techniquedel'extension du voyge, soucid'offrir un maximum de services dans un minimum de temps...). Pour tous ces motifs l'entreprise Rivet, puis l'entreprise Rivet-Hone, représentent une valeur symbolique pour l'histoire touristique au Québec.

C'estce qui a amené le Groupede recherche en patrimoine à entreprendre cette étude et à présenter dans sa version intégrale le texte de l'édition spéciale du numéro Le Touriste 1907. Cette recherche s'inscrit dans un cadre plus étendu qui est celui des rapports entre l'activité touristique et la société québécoise au début du $X X^{*}$ siècle, 1900-1930, environ. Les résultats que nous livrons dans cette publication restent sommaires mais nous apparaissent tout de mểne révélateurs del'importance que nous devons lui accorder. Les hypothèses que nous avons posées au départ demandent encore temps et approfondissement; ainsi reste-t-il un important travail à accomplir afin d'en arriver à une compréhension globale du phénomène touristique dans ce début de siécle et de ses mécanismes partculiers de fonctionnement. Il nous faudra awoir accèsà des sources dedocumentations plus complètes et à caractère économique afin d'y parvenir. Une analyse exhaustuve des contenus descriptifs, publicitaires, commerciaux et iconographiques du Touriste (éditions successives) est en cours et nous entreprenons, parallèlement, l'analyse et l'étude du stouristew de Fraserville afin de déterminer les paradigmes du tourisme régional et international au Québec pour la période qui nousconcerne. Nous rendonsici hommage à la thèse de monsieur Roger Brière qui ouvrait en 1967 les voies de la recherche en histoire du tourisme au Québec. La présente édition de Documents s'adresse à tous les intervenants touristiques et culturels, soucieux de parfaire leur connaissance denotre patrimoinenational. Nous aurons atteint notre objectif actuel si nous réussissons à leur communiquer cet intérët et à

\section{AGENCE DES VOYAGES RIE E T}

Billets de Passage pour tous les Pays, par toutes les Lignes.

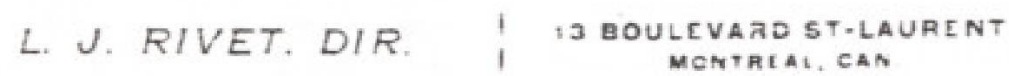

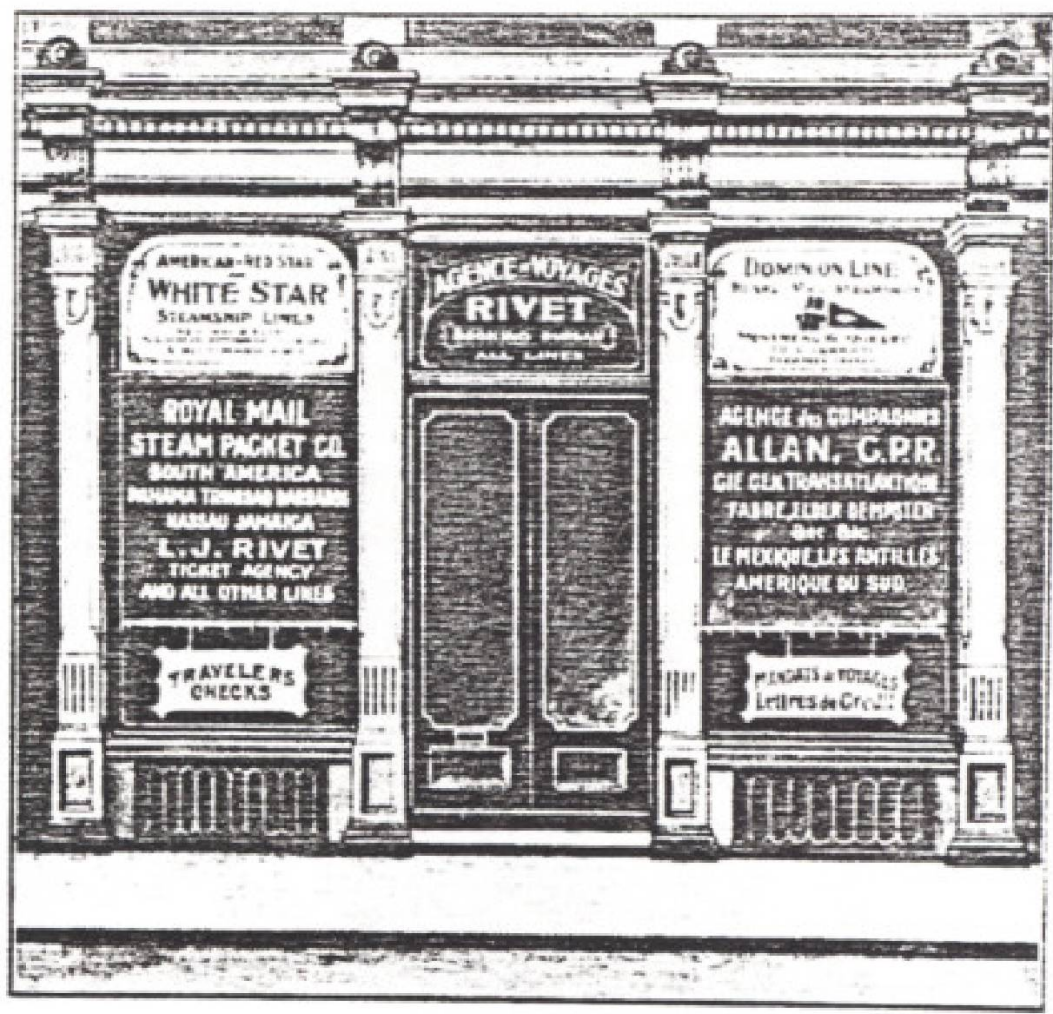

On voit ici la facade de l'Agence de voyage River sur une page de publicité du premier numéro de la revue * Le Touristem, publiée en 1907 a Montréal par Louis Joseph Rivet. Photo tirée de la reproduction de cette revue par le Groupe de recherche en patrimoine de I'UQAM, en 1978, dirigé par P. Mayrand.

les associer à notre enquête sur les origines du tourisme contemporain au Québec.

\section{Première période: les années d'apprentissage, 1875-1902}

La littérature touristique au XIX" siècle: au cours du XIX siècle, un grand nombre de guides touristiques sont publiés à Montréal età Québec. Leurs auteurs ne peuvent être qualifiés de spécialistes en matière de tourisme national et international, car à l'époque, aucune agence, aucune école ne dispense une formation quelconque dans ce domaine. Ce sont alors des chroniqueurs, des journalistes ou, tout simplement, des amateurs qui, comme L.J. Rivet cultivent un goût personnel. D'autre part, on peut supposer que ce dernier a puisé à l'intérieur de ces guides (entre autres, les guides Baedeker, très prisés à l'époque; publicité de ceux-ci à l'intérieur du Touriste 1907). des informations techniques et essentielles à la bonne organisation d'un voyage.

Organisation d'un premier voyage: étant donné qu'il ne connaissait pas à fond les tarifs de tous les lieux qu'il visitait, il pro- 
cédait de la manière suivante: \&Il payait tous les frais encourus durant la journée puis le soir les woyageurs de son groupe lui versaient leur quote-part* (témoignage de monsieur François Hone).

Bureau de l'Agence générale des voyages: 1901-05 c: 87, rue St-Jacques.

$1875 \mathrm{c}$ Naissanceprobable de Louis Joseph Rivet.

$1890 \mathrm{~cm}$ LJ. Rivet exerce la profession de «vendeur et accordeur de pianosw. Il travaille alors, à cette époque, pour la *Compagnie Laffargue Inc,», entreprise de grande envergure ayant son siège social à New York Sa profession, semble-t-il, l'oblige à se déplacer. Cela, uni à un goût personnel des voyages, peutlaisser supposer que L.J. Rivet acquiert durant ces années, et de lui-même, la formation touristique et l'expérience qui lui serviront plus tard, au sein de sa nouvelle agence touristique. * $\mathrm{De}$ retour de son premier voyage en Europe, voyage où il avait minutieusement observé les lieux, les couturnes, les façons d'être, les façons de voyager, les monuments grandioses comme les hôtels confortables, fouillé les bibliothèques et les méthodes de l'agence Cook, il fit nombre de conférences afin de faire partager aux Canadiens de toutes les classes son ardeur voyageuse....

*Voyages d'Europes du *Paris-Canada", Paris, $1^{*}$ octobre 1901

1901-92: Une brochure est publiée à cette époque sous les auspices de la *compagnie de navigation du Richelieu et Ontarios, L.J. Ruvety présentel'itinéraired'un woyage à l'intention des Français invités à venir au Canada à l'occasion de l'exposition de Buffalo. Le voyage est organisé par * La Chambre de Commerce du District de Montréals et par * La Chambre de Commerce Françaises, La brochurementionne également deux succursales, une à Paris, l'autre à New York, de même que l'adresse de son bureau de Montréal, sur la rue StJacques. C'est alors entre les années 1901 02 que l'on peut situer les débuts profes= sionnels de LJ. Rivet dans le domaine touristique. Il est à noter cependant que $\mathrm{L}$.J. Rivet cumule deux emplois et ce, jusqu'en 1911. Le Lovell's Montreal Directory indique à côté de L.J. Rivet: \&Vendeur et accordeur de pianos $*$ et directeur des *Voyages Rivet* (réf.: Lovell's Montreal Directory, 1902).

\section{Deuxième période : publication Le Touriste, 1907-1910}

Publicité-information: $*$ Voir Londres, Paris, Tours, Lourdes, Pau, Bayonne et les Pyrénées dans des conditions confortables sans avoir à se précocuper des mille et un petits ennuis du voyage, toujours si embarrassants pour celui quivisitel'Europe pour la première fois; votre itinéraire fixé, votre chambre d'hôtel et vos voitures retenues d'avance, votre billet de passage et de chemin de fer acheré, votre bagage en sûreté, renseignements fournis, pourboires payés, voilà qui est bien attrayant, sans doute; mais jouir de tous ces avantages dans des conditions de bon marché exceptionnel, c'est toutsimplementl'idéal - or voilà cequ'offrel'Agence générale desvoyages.... Louis Fréchette, LaPresse, 27 juillet 1897.

Bureau de l'Aqence générale des voyages: 1905-07 c: 5, boul. Saint-Laurent.

1907: l'année 1907 marque la première publication de la revue Le Touriste inaugurée et lancếe par L.J. Rivet.

Fait important: L.J. Riveta ếpousé Délima Berthiaume, fille d'un des magnats de l'information journalistique francophone (La Presse). Cette union favorise, sans aucun doute, la publication du Touriste de même qu'un échange de services publicitaires entre $\mathbf{L}$ a Presse et l'Aqence. Cette revue se place à l'avant-garde dans le domaine de la diffusion de l'information touristique.

Elle offre au voyageur:

- des conseils pratques;

- une description succincte mais précise des moyens de transports mis à sa disposition, de même que les durées, tarifs et destinations proposées;

- une présentation des principales villes, régions et pays susceptibles d'enrichir le voyageur, tant sur le plan culturel que naturel;

- des projets d'itinéraires courants (Montréal-Le Havre; MontréalLondres et d'autres plus particuliers (Egypte-Palestine-Haute-Syrie);

- la revue met également à la disposition du voyageur une cribune libre où celui-ci peut alors faire partager aux autres lecteurs ses récits de voyages, ses impressions, etc.
1908 c: réédition de la revue Le Touriste. Le contenu et la publicité sont, dans l'ensemble, semblables au numéro précédent. Il semble, cependant, qu'un certain C.O. Lamontagne aurait eu un rôle à joucr dans la rédaction de certains textes, mais ccla reste à vérifier.

\section{Troisième période: I'Association Jules Hone et Louis Joseph Rivet, 1911-1916}

\section{Information touristique régionale:}

1910: de la ville de Fraserville est lancée une petite publication portant également le nom de Touriste mais n'ayant, semble= $\mathrm{t}$-il, et à première vue, aucune relation avec la revue de L.J. Rivet. Cette revue fait la promotion:

- du tourisme régional (bas du fleuve; Rivière-du-Loup):

- possède deux objectifs: d'une part favoriser la connaissance historique et culturelle de la région; d'autre part, attirer des investissements afin de permettre le développement économique de la région.

Tragédie maritime:

10) avril 1912: naufrage du Titanic. Cet événement a sans doute ralenti momentanément l'activitế touristique.

\section{Déclaration de guerre:}

1914: déclaration de la Première Guerre mondiale. Un pèlerinage organisé par l'Agence Hone et Rivet est rapatrié d'urgence.

Bureaux del'Agence générale des voyages:

1909-12 c: 13 , boul. Saint-Laurent. 1912-21 c: 9, boul. Saint-Laurent.

Il est à noter que tous les bureaux de l'Agence de 1901 à 1921 sont situés dans la zone portuaire.

1911-12: association de LJ. Rivet et de Jules Hone, l'Agence prendra alors le nom de Hone et Rivet.

Jules Hone débute au Canadien Pacifique en 1903, à Québec. Il deviendra par la suité directeur du Grand Tronc. Il quitte alors un emploi assuré et se lance dans une entreprise toute nouvelle: agent de voyages. Au service du Canadien Pacifique, J. 
Hone a acquis une expérience évidente: il traçait pour ses clients des projets d'itinéraires, de lieux à visiter, etc. Etant donné que cette manière de procéder lui faisait déjà toucher des commissions appréciables, devenir directeur associédel'Agence Rivet, lui permettait de réaliser alors des bénéfices plus substantiels.

réf:: Témoignage vivant de monsieur François Hone.

D'autre part, l'agence Rivet permettait à J. Hone, non plus simplement, l'accessibilité à un tourisme régional, mais bien à un tourisme international.

$1912 \mathrm{c}$ date de la première publication du Touriste durant l'association Hone et Rivet.

\section{À l'intérieur de la revue:}

- une grande place est accordée à la publicité (rentabilité de la revue);

- les textes descriptifs sont en tuès petit nombre si l'on compare cette revue avec celle de 1907;

- le format est rectangulaire, donc plus pratique; la page couverture de cette revue est la même que celle du Touriste de 1907: La voyageruse de la belle épogue;

- if est fait mention de deux bureaux Hone et Rivet. L'un à Montréal, l'autre à Québec;

- les succursales de Paris et de New York ne sont pas mentionnées.

1914: à la veille de la Première Guerre mondiale, une secondeéditiondu Touriste est lancée:

- le contenu est, dans l'ensemble, semblable au numéro précédent;

- encore une fois, une grande place est accordée à la publicité;

- dans ce numéro on mentionne une succursaleà Paris. Celle de New York semble ne plus exister;

- la page couverture symbolise le progrès, donc la rapidité dans le domaine descommunications (tuain, paquebot, avion courrier).

L'année 1914 de mềme que celles qui suivront jusqu"à la fin de la guerre marquent une période sombre pour le tourisme. Il va sans dire que les affaires périclitent et qu'il ne peut être question devoyagesd'agrément en Europe. Cependant, l'Agence conti- nuera d'offrir ses services durant toutes les années de guerre.

Quatrième période: fin de la carrière de L.J. Rivet dans le domaine du tourisme et promotion honorifique à l'étranger, 1917-1932

Résidence de L.J. Rivet:

1897-1901 c: 418, rue Rachel (L.J. Rivet vit alors avec son père, Alfred Napoléon Rivet, physicien et professeur à l'Université Laval).

1901-06 c 140, rue St-Urbain (cette habitation semble être un immeuble à plusieurs logements).

$1906-08 \mathrm{c:} 465$, rue St-Urbain (maison à plusieurs logements).

$1908-10 \mathrm{c:} 467$, rue St-Urbain (maison à plusieurs logements).

1910 c: 715 , rue St-Urbain.

1911 c: 711, rue St-Urbain.

1912 c: 240, Mc Dougall, Outremont.

1913-21 c. 595, rue St-Denis.

1917-18 c: cette date marque la fin de l'association Hone et Rivet. L.J. Rivet abandonne complètement sa carrière en tourisme et quitte le Canada quelques années plus tard (nous ne connaissons pas la date exacte de son départ). Il séjournera à Lourdes pour finalements'installer à Rome où il deviendra Camerier secret du pape Pie XI. Jules Hone assume donc seul la direction de l'Agence qui prend alors le nom de: Les Agences de vayages f. Hone.

$1918-19 \mathrm{c}$ avec la fin de la guerre et en vue de relancer le tourisme et les voyages qui, peu à peu, reprennent vie, J. Hone lance la troisième édition du Touriste.

Il n'est fait mention que d'un seul bureau, celui de Montréal, mais il conserve toujours la succursale de Paris.

La page couverture de la revue est la même que celle de 1914.

1932: décès de J.L. Rivet.

1958: décés de Jules Hone.

1929-72: suite de l'Agence assurée par François Hone. $f$

\section{NOTES}

(1) De telles recherches n'auraient pu aboutir sans l'acharnement de Anne-Marie Fougere, recherchiste.

(2) Ces buénements, réalisés sous la forme de mises en scene thematiques et historiques vivantes,
Iutent présentés a l'Hótel Méridien et au Centre Sheraton. Ils furent intertompus par la dis sociation de l'equipe.

BIBUOGRAPHIE

BAIEAE, Foger, Gbographie du tourisme au Oubbec, these de doctorat, geographie, Universile de Montreal, 1967, 348 pages len particulier le chapitre II: Le XIX* sibde et la premiere moitie du XX* sidele).

HONE, Franpois, Un sibcle ot demi de documente historiques. La facelnante pethte histoire de nou familles Julea Hone - Antoine Gurin Lajoie. La or ande histoir e tr be weuvent dramatique de rAgence de vorage, vol. 1.

LORD. Francine, Lee guides touriatiques du Oubbec. wu X|X* wibele, Bibliotheqque nationale du Québec, 1976, catalogue d'expostion.

FRAPPIER, Monique, HEAP, Marguaret ef Jean-Claude ROBERT, Montrial, les riteits de voyages: bFbliographle, rapport et travaux 1973-1975, departement d'histoire, UOAM-GRSM.

\section{SOURCES DOCUMENT AIRES:}

Fonds prives: François Hone

Lovell's Montreal Directory: 1897-1921

Palais de justice de Montréal: archives

Guides Baedeker:

- Nord de la France., Paris, 1884

- LLe midi de la Francer, Paris, 1885

- eltalies. Paris, 1890

- Ittalie méridionale. Paris, 1890

- Italie septentrionalem, Paris, 1992

- Italie centralen, Paris, 1894

- Autinche-Hongrie. Paris, 1911 